\title{
Boundary behaviour of modes of a Dirichlet Laplacian
}

\author{
DMITRII KOUZNETSOV†t* and JEROME V. MOLONEY† \\ $\uparrow$ Arizona Center for Mathematical Sciences, Department of \\ Mathematics, University of Arizona, 617 N. Santa Rita, \\ Tucson, AZ 85721, USA \\ Institute for Laser Sciences, University of Electro-Communications, \\ 1-5-1 Chofugaoka, Chofu, Tokyo 182-8585, Japan; \\ e-mail: dima@ils.uec.ac.jp
}

(Received 5 July 2003; revision received 25 September 2003)

\begin{abstract}
The problem of uncoupled modes of the pump in a double-clad fibre amplifier is analysed. As long as such modes avoid the core of the fibre, we consider the Dirichlet Laplacian problem which neglects the core. In this approach, the boundary of the cladding is treated as an ideal mirror. The laws of conservation established for the paraxial equation of diffraction give certain integral relations for the derivatives of modes at the boundary. Such relations are formulated as theorems. These theorems show ways to non-traditional design of single-mode fibre amplifiers with a multimode pump. In particular, the conservation of momentum can be applied to the design of the slab-pumped fibre amplifier. The conservation of angular momentum predicts high efficiency of coupling of a pump into a doped core embedded in a spiral-shaped doubleclad fibre. Such predictions agree with results of numerical experiments published recently and allow a geometric optics interpretation.
\end{abstract}

\section{Introduction}

The most practical application of the Dirichlet Laplacian (DL) deals with paraxial optical waveguides and optical fibres. Double-clad fibre amplifiers convert the partially coherent light from cheap and efficient semiconductor lasers into single mode radiation. In such an amplifier, the multimode pump propagates mainly in the cladding. This cladding should not be too small in order to allow the coupling to the source of the pump. For good performance of such an amplifier, all the modes of the pump should be effectively absorbed in the core. The core provides for discrimination of modes of the signal; the core cannot be too large, unless the tolerance interval of the step of the index of refraction becomes very small. The design of the inner cladding of the double-clad fibre amplifier should provide the effective coupling of most of the pump into the relatively small core. The coupling of the pump cladding modes to the core is especially poor in the case of a fibre with circular symmetry [1-3]; most of the modes are unusable: they avoid the core. In the approximation of geometrical optics [3], most of the rays avoid the core in such a fibre. Various shapes of double-clad fibres were used/simulated in order to improve the coupling of the pump into the core [2-12]: off-set [2], rectangular [2, 6], smooth rectangular [4], smooth hexagon [4], spiral [4, 5],

* Author for correspondence.

Journal of Modern Optics ISSN 0950-0340 print/ISSN 1362-3044 online (C) 2004 Taylor \& Francis Ltd http://www.tandf.co.uk/journals

DOI: $10.1080 / 0950034042000200533$ 
D-shape [6-8], truncated rectangle [6], 2-truncation circle [6, 7, 9], pseudorectangle [6,9], stadium [9], smooth D-shaped [10], decagon [11] and 'flower' $[6,12]$. The variety of shapes analysed and tested recently indicates that the choice of shape of cladding is an important and non-trivial problem. The circular off-set fibre is much better than the symmetric one. Rectangular, D-shaped and many other claddings provide a slightly better coupling than the circular off-set fibre does. The double-cut circular cladding [6-9] and the spiral cladding [4, 5] seem to be the most efficient configurations, according to the numerical analysis. Here we present some analytical results, which explain the advantages of the spiral-shape cladding $[4,5]$ in terms of modes of the DL.

The modes of double-clad fibres are not the same as modes of the DL; the core disturbs modes. Nevertheless, consideration of the DL improves our understanding of the behaviour of the pump. The large inner cladding may support both coupled and uncoupled pump modes. Coupled modes are absorbed by the core, so, we do not worry about them. As for the uncoupled modes, they avoid the core; so, they should be similar to modes of the core-less waveguide. For this reason, here we consider modes of the DL.

A paraxial mode in an idealized curvilinear waveguide relates to the solution of the Helmholtz equation

$$
(\Delta+\lambda) \Psi=0,
$$

for some real $\lambda$ in some domain $\mathcal{G}$ of the two-dimensional Euclidean space and boundary condition $\Psi=0$ on $\partial \mathcal{G}$. This refers to the Dirichlet Laplacian (DL) problem $[13,14]$. The real eigenfunction $\Psi$ satisfying (1) and the boundary condition should be treated as a mode of the DL.

Some theoretical results about modes of the DL should be mentioned. Theoretical bounds on the set of eigenvalues $\lambda_{n}, n=1,2, \ldots$ were reported [13]. The properties of the nodal surfaces (lines) of first modes have been identified [14], although the cost of the calculation seems to increase rapidly with mode number. For analysis of the highest modes, quasi-classical [15] and statistical approaches [16] have been employed. Such analysis shows the essentially non-uniform statistics of the distribution of the amplitude of the modes. The modes show localization or scarring $[17,18]$ around periodic orbits, even if such trajectories are classically unstable. Lines of localization of amplitude of modes are called 'scars'. Such localization or scarring is especially strong for symmetric domains, for example, the stadium billiard [19]. For a given mode of the classically chaotic system, the distribution of intensity shows no evidence of uniformly filling the domain.

Recently, a completely asymmetric 'spiral-shaped' inner cladding was analysed numerically using the beam propagation method [4] as well as a mode expansion technique [5]. It was shown that a small asymmetric distortion of an otherwise circular cladding significantly improves the efficiency of coupling of the incoherent light of the pumped cladding into the core. Such an improvement is comparable to or even greater than the improvement due to a strong double-cut of the cladding [6-9]. The spiral is not a closed curve so, the spiral-shaped boundary of the region must have a strong local deformation somewhere. Overlap of modes with the relatively small core is especially good, when this core is located close to this region of deformation. Here we present a theoretical interpretation of these observations. 
In this paper, we analyse the boundary behaviour of the modes of a region $\mathcal{G}$ of a general shape. Theorem 3 below indicates that the derivative of the mode cannot be zero in the region of the deformation. The spiral shape has no need to eliminate the scars of the modes. If a mode has strong scars and is very small in most of the domain, then some of these scars must be close to the region of deformation. The core placed in the vicinity will effectively absorb all the modes of the pump.

The idea is to use the conservation laws established for paraxial propagation in wave optics $[20,21]$. The assumption of a mode with trivial (exponential) dependence on the longitudinal coordinate gives the relations to be satisfied at the edge of the domain. The results will involve integrals of the derivatives of the mode at this boundary. While the modes vanish at the boundary, the resulting expressions will be integrals of bilinear forms of derivatives of the modes. For simplicity, here we apply only two laws of conservation (conservation of momentum and angular momentum), and present the deduction for the steady-state solutions of the equation of diffraction, which corresponds to the DL.

\section{Notations and background}

For simplicity of deduction, we use compact notations. Let

$$
\begin{aligned}
x & =\left\{x_{2}, x_{2}\right\} \in \mathbb{R}^{2}, \quad \Psi=\Psi(x) \in \mathbb{R}, \\
\Psi_{; \alpha} & =\partial \Psi / \partial x_{\alpha},
\end{aligned}
$$

where Greek letters in the subscript take values 1 or 2 . Then, the modulus of gradient of the mode can be written as follow:

$$
\Psi^{\prime}=\left|\nabla \Psi\left(x_{1}, x_{2}\right)\right|=\left(\Psi_{; 1}^{2}+\Psi_{; 2}^{2}\right)^{1 / 2}=\left(\Psi_{; \alpha} \Psi_{; \alpha}\right)^{1 / 2} .
$$

We assume summation with respect to repeating subscripts. Using this notation, the DL equation can be written as follows:

$$
\begin{aligned}
\Psi_{; \alpha ; \alpha}+\lambda \Psi & =0, \quad x \in \mathcal{G} \subset \mathbb{R}^{2}, \\
\Psi & =0, \quad x \in \partial \mathcal{G} .
\end{aligned}
$$

For the formulation of theorems, we need two additional objects:

$$
\begin{aligned}
W_{\alpha, \beta} & =\Psi_{; \alpha} \Psi_{; \beta}-\Psi_{; \alpha ; \beta} \Psi, \\
M_{\beta} & =\varepsilon_{\alpha, \gamma} x_{\alpha} W_{\gamma, \beta},
\end{aligned}
$$

where $\varepsilon_{1,1}=\varepsilon_{2,2}=0, \varepsilon_{1,2}=1, \varepsilon_{2,1}=-1$. Physically, $W$ corresponds to the flux of the momentum and $M$ corresponds to the flux of angular momentum of the corresponding dynamical system. However, for the real mode, we have no need to specify complex conjugation, as in the case of propagation considered in $[20,21]$. For our case, the local conservation of momentum and angular momentum can be formulated as follows:

$$
\begin{aligned}
W_{\alpha, \beta ; \beta} & =0, \\
M_{\alpha ; \alpha} & =0 .
\end{aligned}
$$

for $x \in \mathcal{G}$. For verification, one can substitute definition (7) into the left-hand sides 
of equation (9) and use equation (5); this gives zero. Then one can substitute definition (8) into the left-hand side of (10) and use equation (9) and then the symmetry $W_{\alpha, \beta}=W_{\beta, \alpha}$.

Also, we need Gauss's theorem: for any vectorial field $F=F(x) \in \mathbb{R}^{2}$, differentiable within $\mathcal{G}$,

$$
\int_{x \in \mathcal{G}} F_{\alpha ; \alpha} \mathrm{d}^{2} x=\oint_{x \in \partial \mathcal{G}} F_{\alpha} \mathrm{d} n_{\alpha},
$$

where

$$
\mathrm{d} n_{\alpha}=\varepsilon_{\alpha, \beta} \mathrm{d} x_{\beta}
$$

is element of the boundary of the domain $\mathcal{G}$.

One additional property of the derivative of the mode at the boundary should be mentioned. Due to equation (3), the gradient of the mode is orthogonal to the element $\mathrm{d} x$ of the contour surrounding $\mathcal{G}$, and parallel (or anti-parallel) to the element of the boundary $\mathrm{d} n$. Therefore the derivative of the mode at the boundary can be expressed as follows:

$$
\Psi_{; \alpha}= \pm \Psi^{\prime} \frac{\mathrm{d} n_{\alpha}}{\left(\mathrm{d} n_{\beta} \mathrm{d} n_{\beta}\right)^{1 / 2}}, \quad x \in \partial \mathcal{G} ;
$$

the upper sign corresponds to the parallel orientation of these vectors and the lower sign corresponds to the anti-parallel case. Therefore the choice of the signum is the same for both components of the gradient and $\Psi^{\prime}$ is the modulus of the normal derivative of the mode at the boundary.

In the following section, Gauss's theorem (11) is used twice: first, for $F_{\alpha}=W_{\alpha, \beta}$ at $\beta=1,2$ and then for $F_{\alpha}=M_{\alpha}$. Equations (4) and (13) allow the representation of the results in compact and elegant form.

\section{Derivatives of the mode at the boundary}

Physically, the square of the normal derivative of the field at the boundary can be interpreted as pressure. For a stationary field, the corresponding force should be equal to zero, as well as the angular momentum. However, this conclusion follows also from equations (5) and (6). In this section, we present the analytical proof.

Theorem 1. Under condition (5) and (6), for $\alpha=1,2$,

$$
\oint_{x \in \partial \mathcal{G}}\left(\Psi^{\prime}\right)^{2} \mathrm{~d} x_{\alpha}=0 .
$$

Proof. Using conservation law (9), Gauss's formula (11), definition (7), and boundary condition (6) sequentially, we obtain: 


$$
\begin{aligned}
& 0=\int_{x \in \mathcal{G}} W_{\alpha, \beta ; \beta} \mathrm{d}^{2} x=\oint_{x \in \partial \mathcal{G}} W_{\alpha, \beta} \mathrm{d} n_{\beta}, \\
& 0=\oint_{x \in \partial \mathcal{G}}\left(\Psi_{; \alpha} \Psi_{; \beta}-\Psi_{; \alpha ; \beta} \Psi\right) \mathrm{d} n_{\beta}, \\
& 0=\oint_{x \in \partial \mathcal{G}}\left( \pm \Psi^{\prime}\right) \frac{\mathrm{d} n_{\alpha}}{\left(\mathrm{d} n_{\gamma} \mathrm{d} n_{\gamma}\right)^{1 / 2}}\left( \pm \Psi^{\prime}\right) \frac{\mathrm{d} n_{\beta}}{\left(\mathrm{d} n_{\gamma} \mathrm{d} n_{\gamma}\right)^{1 / 2}} \mathrm{~d} n_{\beta}, \\
& 0=\oint_{x \in \mathcal{H}}\left(\Psi^{\prime}\right)^{2} \frac{\mathrm{d} n_{\alpha} \mathrm{d} n_{\beta}}{\mathrm{d} n_{\gamma} \mathrm{d} n_{\gamma}} \mathrm{d} n_{\beta}=\oint_{x \in \partial \mathcal{G}}\left(\Psi^{\prime}\right)^{2} \mathrm{~d} n_{\alpha} .
\end{aligned}
$$

Multiplying the last expression by $\varepsilon_{\alpha, \beta}$, summing by $\alpha$ and applying (12), we get statement (14) of the theorem. (End of proof)

Theorem 2. Under conditions (5) and (6),

$$
\oint_{x \in \mathcal{G}}\left(\Psi^{\prime}\right)^{2} x_{\alpha} \mathrm{d} x_{\alpha}=0 .
$$

Proof. Using conservation law (10), Gauss's Theorem (11), definition (8), boundary condition (6) and equation (13) sequentially, we get:

$$
\begin{aligned}
& 0=\int_{x \in \mathcal{G}} M_{\alpha, \alpha} \mathrm{d}^{2} x=\oint_{x \in \partial \mathcal{G}} M_{\alpha} \mathrm{d} n_{\alpha}, \\
& 0=\oint_{x \in \partial \mathcal{G}} \varepsilon_{\beta, \gamma} x_{\beta}\left(\Psi_{; \alpha} \Psi_{; \gamma}-\Psi_{; \alpha ; \gamma} \Psi\right) \mathrm{d} n_{\alpha} \\
& 0=\oint_{x \in \partial \mathcal{G}} \varepsilon_{\beta, \gamma} x_{\beta}\left( \pm \Psi^{\prime}\right) \frac{\mathrm{d} n_{\alpha}}{\left(\mathrm{d} n_{\mu} \mathrm{d} n_{\mu}\right)^{1 / 2}}\left( \pm \Psi^{\prime}\right) \frac{\mathrm{d} n_{\gamma}}{\left(\mathrm{d} n_{\nu} \mathrm{d} n_{\nu}\right)^{1 / 2}} \mathrm{~d} n_{\alpha}, \\
& 0=\oint_{x \in \partial \mathcal{G}} \varepsilon_{\beta, \gamma} x_{\beta}\left(\Psi^{\prime}\right)^{2} \frac{\mathrm{d} n_{\alpha} \mathrm{d} n_{\gamma}}{\mathrm{d} n_{\nu} \mathrm{d} n_{\nu}} \mathrm{d} n_{\alpha}=\oint_{x \in \partial \mathcal{G}}\left(\Psi^{\prime}\right)^{2} \varepsilon_{\beta, \gamma} x_{\beta} \mathrm{d} n_{\gamma} .
\end{aligned}
$$

Using the relation $\varepsilon_{\beta, \gamma} \mathrm{d} n_{\gamma}=-\mathrm{d} x_{\beta}$, we get statement (19) of the theorem. (End of proof)

Consider a special case. Let the boundary $\partial \mathcal{G}$ be parametrized in cylindrical coordinates:

$$
x_{1}=R(\phi) \cos (\phi), \quad x_{2}=R(\phi) \sin (\phi) ; \quad \phi \in[-\pi, \pi]
$$

where $R=R(\phi)$ is a smooth function and $R(-\pi)=R(\pi)$. Using these notations we formulate

Theorem 3. For the function $\Psi$ satisfying (5) and (6) with the parametrization (24),

$$
\int_{-\pi}^{\pi}\left(\Psi^{\prime}\right)^{2} R R^{\prime} \mathrm{d} \phi
$$

where $R^{\prime}=\mathrm{d} R(\phi) / \mathrm{d} \phi$.

Proof. Let $C=\cos (\phi), S=\sin (\phi)$. Then at $x \in \partial G$, we have $x_{1}=R C, x_{2}=R S$; $\mathrm{d} x_{1}=\left(R^{\prime} C-R S\right) \mathrm{d} \phi, \mathrm{d} x_{2}=\left(R^{\prime} S+R C\right) \mathrm{d} \phi$. Using Theorem 2, we have 


$$
0=\oint\left(\psi^{\prime}\right)^{2} x_{\alpha} \mathrm{d} x_{\alpha}=\int_{-\pi}^{\pi}\left(\psi^{\prime}\right)^{2}\left(R C\left(R^{\prime} C-R S\right)+R S\left(R^{\prime} S+R C\right)\right) \mathrm{d} \phi .
$$

Since $C^{2}+S^{2}=1$, we get statement (25) of the theorem. (End of proof)

The theorems of this section give certain limits of the variety of modes of DL. From Theorem 1 we see that the mode cannot have a strong derivative at the left boundary of the domain and small derivative at the right boundary, as the total integral of the flux of the momentum should be zero. Theorem 2 is important for a domain $\mathcal{G}$ with slightly broken circular symmetry. For this case, the parametrization (24) is valid and we can use Theorem 3 instead, which is a special case of Theorem 2. Examples of use of Theorems 1 and 3 are considered in the following section.

It should be noted that similar integral relations take place for other conservation laws mentioned in $[20,21]$. We do not know their direct application to optics of waveguides, therefore we do not discuss them here.

\section{Application of theorems 1 and 3}

Example of use of Theorem 1. Consider a waveguide of L-shaped cross-section (figure 1). Such a configuration can correspond to a specific realization of a doubleclad fibre, where the left thick part is designed for the propagation and amplification of a signal, and the long right part is designed for the propagation of a multi-mode pump from a semiconductor laser. The light output of the latter is highly coherent in the $x_{2}$ direction and has many modes in the $x_{1}$ direction. The left side corresponds to the doped core and is adjusted for single-mode propagation at the signal wavelength. Highly excited modes in the thin waveguide have large $x_{1}$ derivative in the right-hand guide. This relates to chaotic bursts from the pump laser along the $x_{1}$ direction. Then, according to Theorem 1, the mode must have a significant $x_{1}$ derivative in the left-hand side of the waveguide (core) also. Such a requirement is important for the design of the delivery of the multimode pump to the fibre amplifiers. The mode cannot exhibit scars, localized only in the narrow part of the waveguide. The highest modes have a strong derivative at the righthand side of the slab. Therefore these modes should show especially good overlap with the left-hand side. This gives a hint for a new design of compact fibre amplifiers with a partially coherent pump considered in [22].

Example of use of Theorem 3. Consider the case, when the domain $\mathcal{G}$ is defined in polar coordinates with equation (24). Let $R^{\prime}>0$ at $-\phi_{0}<\phi<\phi_{0}$, where $\phi_{0}>0$ is a parameter slightly smaller than $\pi$. Such a parametrization leads to a spiral contour (figure 2) strongly deformed at the left-hand side. The integral in (25) can

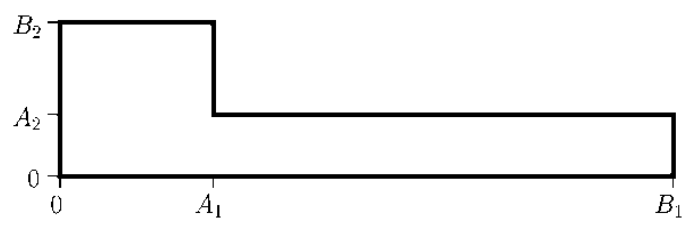

Figure 1. Example of the application of Theorem 1: $\int_{0}^{B_{2}} \Psi_{; 1}(0, y)^{2} \mathrm{~d} y=\int_{A_{2}}^{B_{2}} \Psi_{; 1}\left(A_{1}, y\right)^{2} \mathrm{~d} y+\int_{0}^{A_{2}} \Psi_{; 1}\left(B_{1}, y\right)^{2} \mathrm{~d} y$. 


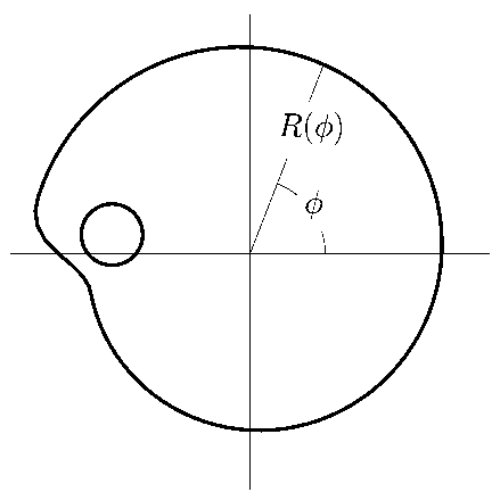

Figure 2. Example of an application of Theorems 2 and 3: spiral-shaped domain. The

be represented as the sum of two integrals: one over the region where $R^{\prime}>0$ and another over the region where $R^{\prime}<0$. While the sum of these two integrals is equal to zero, the mode cannot exhibit scars approaching the boundary only at $|\phi|<\phi_{0}$; some scars of each mode approach the region of deformation too. Therefore, such a domain cannot have modes avoiding the region of deformation. This property of the domain was used to predict the uni-directional output of the spiral-shaped micro-disc laser, reported recently [23].

Consider the double-clad fibre amplifier with cladding parametrized with function $R$ specified above. No one mode can exhibit strong scars avoiding the region of deformation. Let us place the core there (figure 2); then all the modes of the pump will have strong overlapping with the core. This conclusion agrees with the analysis of modes described in [5]; the core will be effectively pumped, it will use all the modes of the cladding. This conclusion agrees with numerical simulations of propagation of a paraxial pump described in [4]. The fibre with such a spiral cladding seems to be chaotic, but it is important to place the core in the vicinity of deformation, otherwise the efficiency of use of the pump in such a fibre will not be better than that of D-shaped or other chaotic fibres [7].

\section{Analogy with geometrical optics}

Theorems 1-3 have an analogy in geometrical optics. Consider a ray which has reflected from the right side of the waveguide of cross-section of figure 1 or figure 2. This ray should unavoidably be reflected from the left side. In the case of the L-waveguide (figure 1), the frequency of reflections of a ray from the right walls should be equal to that of reflections from the left walls.

Consider the spiral-shaped waveguide (figure 2). The helicity of a ray can be defined as the distance at which the ray passes the optical axes, counted with positive sign if it goes counter clockwise and with negative sign if it goes clockwise. This helicity increases at each reflection from the spiral part of the boundary, until occasional reflection form the deformation, or until the helicity becomes of order $R$. The helicity cannot become larger than $R$, so, the ray hits the region of deformation in order to spend its helicity. Then it passes through the core placed in the vicinity of deformation. Therefore all the rays participate in the pumping of the core. To our knowledge, it is the only type of wide cladding which does not allow any ray to avoid the core. 


\section{Conclusions}

Conservation of momentum and angular momentum leads to Theorems 1-3 for the modes of the DL. These theorems put certain restrictions on the distribution of the normal derivatives of the modes. Theorem 1 indicates that the high modes of an L-shaped waveguide (figure 1) have good overlap with the left-hand part of the waveguide. This gives a hint for the new design of delivery of a pump to fibre amplifiers [22]. Theorems 2 and 3 reveal the specific property of the spiral-shaped domain. All the modes have a significant value in the vicinity of the deformation. The spiral-shaped double-clad fibre amplifier should have especially good coupling of power of the pump into the core placed in the vicinity of deformation. This conclusion agrees with results of numerical analysis [4, 5], and allows for an explicit geometric optics interpretation.

\section{Acknowledgments}

The authors are grateful to Miroslav Kolesik, Robert Indik, Moysey Brio and other colleagues from ACMS for their help and discussions. This work was supported by US Air Office of Scientific Research grant F49620-02-1-0380.

\section{References}

[1] Bedö, S., Lüthy, W., and Weber, H. P., 1993, Optics Commun., 99, 331.

[2] Liu, A., and Ueda, K., 1996, Optics Commun., 132, 511.

[3] Kouznetsov, D., Moloney, J. V., and Wright, E. M., 2001, J. opt. Soc. Am. B, $18,743$.

[4] Kouznetsov, D., and Moloney, J. V., 2002, J. opt. Soc. Am. B, 19, 1259.

[5] Kouznetsov, D., and Moloney, J. V., 2002, J. opt. Soc. Am. B, 19, 1304.

[6] Leproux, P., Février, S., Doya, V., Roy, P., and Pagnoux, D., 2001, Opt. Fiber Technol., 6, 324.

[7] Doya, V., Legrand, O., Mortessagne, F., and Miniatura, C., 2002, Phys. Rev. E, 65, 056223 .

[8] Doya, V., Legrand, O., and Mortessagne, F., 2002, Optics Lett., 26, 872.

[9] Leproux, P., Doya, V., Roy, P., Pagnoux, D., Mortessagne, F., and Legrand, O., 2003, Optics Commun., 218, 249.

[10] Renner, R., Kehrli, M., Lüthy, W., and Weber, H. P., 2003, Laser Phys., 13, 232.

[11] Young, D., and Roychoudhuri, C., 2003, Optics Express, 11, 830.

[12] Tsang, Y. H., King, T. A., Thomas, T., Udell, C., and Pierce, M. C., 2003, Optics Commun., 215, 381.

[13] Ashbaugh, M. S., and Benguria, R. D., 1994, Proc. Am. math. Soc., 121, 145.

[14] Fournais, S., 2001, J. differ. Equations, 173, 145.

[15] Zaitsev, O., Narevich, R., and Prange, R. E., 2001, Found. Phys., 31, 7.

[16] Li, B. W., and Rouben, D., 2001, J. Phys. A, 34,7381.

[17] Kaplan, L., 1998, Phys. Rev. Lett., 80, 2582.

[18] Bies, W. E., Kaplan, L., Haggerry, M. R., and Heller, E. J., 2001, Phys. Rev. E, 63, 066214.

[19] Vergini, E., and Saraceno, M., 1995, Phys. Rev. E, 52, 2204.

[20] Kuznetsov, E. A., and Turitzin, S. K., 1985, Phys. Lett. A, 112, 273.

[21] Kuznetsov, D., 1993, Nonlinear and Quantum Phenomena in Nonequilibrium Media, edited by V. A. Shcheglov (Nova Science Publishers).

[22] Kouznetsov, D., and Moloney, J. V., 2003, IEEE J. quantum Electron., 39, 1452.

[23] Chern, G. D., Tureci, H. E., Stone, A. D., Chang, R. K., Kneissl, M., and Johnson, N. M., 2003, Appl. Phys. Lett., 83, 1710. 